\title{
Hybrid Synchronization of Fractional Order Chaotic Systems
}

\author{
Xue-feng Liang, Li-xin Yang ${ }^{a}$ \\ School of Mathematics and statistics, Tianshui Normal University, Tianshui, China \\ ay0931l@163.com
}

Keywords: Modified adaptive hybrid projective synchronization; Fractional-order chaotic systems; Scaling factors; Adaptive control

\begin{abstract}
This paper studies the modified hybrid projective synchronization of fractional order chaotic systems, which generalizes many various synchronization forms. We firstly present this kind synchronization that the response and drive systems have scaling factors at the same time. In addition, based on the stability analysis of fractional order systems and adaptive control technique, a suitable controller and parameter update law can be designed to achieve the modified hybrid projective synchronization for uncertain fractional-order chaotic systems.
\end{abstract}

\section{Introduction}

Recently, synchronization of chaotic fractional-order differential systems have received a significant attention among scientists from various different fields [1]. On one hand, inspired by the pioneering work in 1990 [2], synchronization has attracted increasing attention due to its potential applications in secure communication and signal processing etc, on the other hand, fractional order derivatives provide an excellent instrument for the description of memory properties of various materials and processes. At the same time, it was proved that many fractional-order systems behave chaotically, such as fractional-order Lü system [3], fractional-order Chua's circuit [4] and fractional-order unified system [5]. Among all kinds of chaos synchronization, projective synchronization is the most noticeable one because of its proportional feature [6]. How to effectively realize the modified hybrid projective of two fractional-order chaotic systems with unknown parameters is an important problem for both the theoretical research and practical applications.

\section{Adaptive modified hybrid projective synchronization}

Fractional-order Chen system is described as follows:

$$
\left\{\begin{array}{l}
D_{*}^{q} X_{1}=a\left(x_{2}-x_{1}\right) \\
D_{*}^{q} X_{2}=(c-a) x_{1}-x_{1} x_{3}+c x_{2} \\
D_{*}^{q} x_{3}=x_{1} x_{2}-b x_{3},
\end{array}\right.
$$

When parameters are set by default as $a=35, b=3, c=28$, and $q=0.9$, system (1) exhibits chaotic behaviors as shown in Fig.1(a). The fractional-order Lü system is described by the following state equation:

$$
\left\{\begin{array}{l}
D_{*}^{q} y_{1}=d\left(y_{2}-y_{1}\right) \\
D_{*}^{q} y_{2}=-y_{1} y_{3}+h y_{2} \\
D_{*}^{q} y_{3}=y_{1} y_{2}-f y_{3},
\end{array}\right.
$$

Fig.1(b) displays the chaotic attractor of the fractional-order Lü chaotic system.

The work was supported by Tianshui Normal University “QingLan” Talent Engineering Funds. 

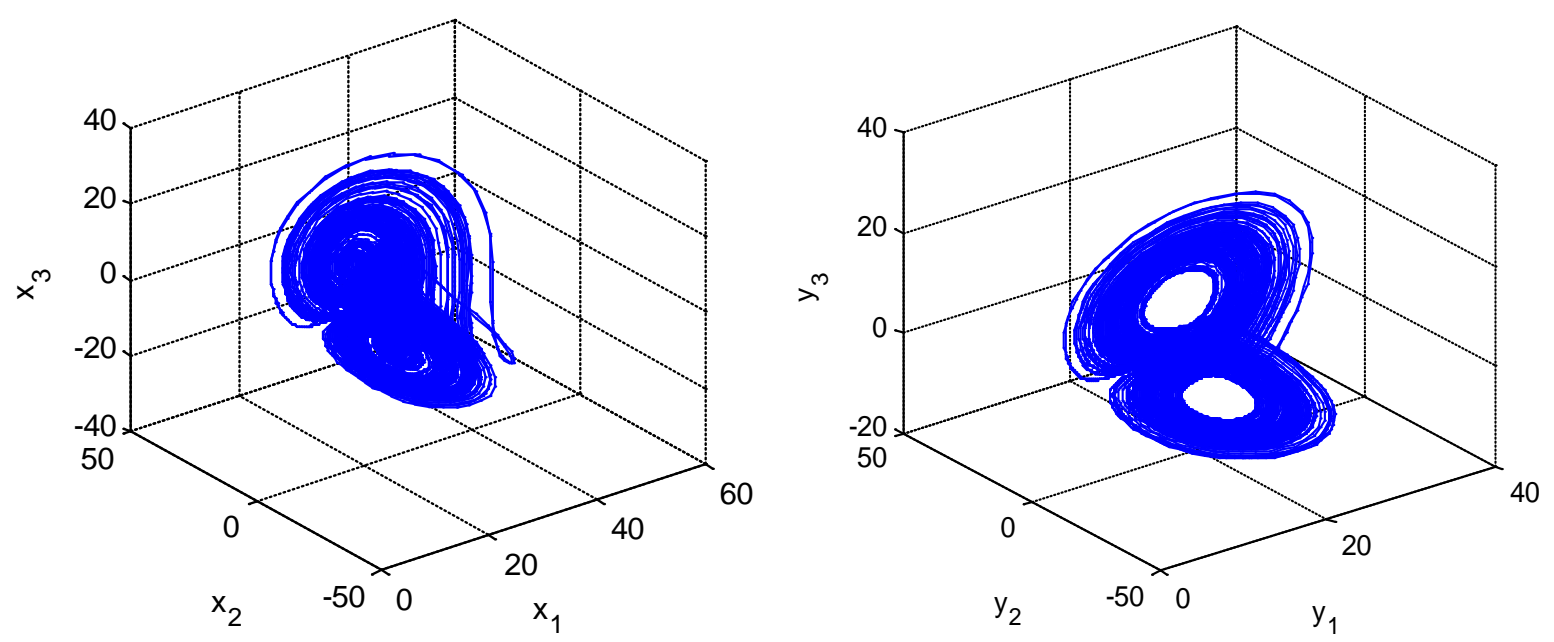

Fig.1 Phase portrait of system (a) Chen system with $q=0.9$. (b) Lu system with $q=0.9$.

We assume that fractional-order chaotic Chen system with unknown parameters is the drive system and the response Lu system is given by:

$$
\left\{\begin{array}{l}
D_{*}^{q} y_{1}(t)=d\left(y_{2}-y_{1}\right)+u_{1} \\
D_{*}^{q} y_{2}(t)=-y_{1} y_{3}+h y_{2}+u_{2} \\
D_{*}^{q} y_{3}(t)=y_{1} y_{2}-f y_{3}+u_{3}
\end{array}\right.
$$

Denote the state errors as $e_{1}=\alpha_{1} y_{1}-\beta_{1} x_{1}, e_{2}=\alpha_{2} y_{2}-\beta_{2} x_{2}, e_{3}=\alpha_{3} y_{3}-\beta_{3} x_{3}$.

It follows from (1)-(3), Then we have the following error dynamical system

$$
\left\{\begin{array}{l}
D_{*}^{q} e_{1}(t)=\alpha_{1}\left[d\left(y_{2}-y_{1}\right)+u_{1}\right]-\beta_{1}\left[a\left(x_{2}-x_{1}\right)\right] \\
D_{*}^{q} e_{2}(t)=\alpha_{2}\left[-y_{1} y_{3}+h y_{2}+u_{2}\right]-\beta_{2}\left[(c-a) x_{1}-x_{1} x_{3}+c x_{2}\right] \\
D_{*}^{q} e_{3}(t)=\alpha_{3}\left[y_{1} y_{2}-f y_{3}+u_{3}\right]-\beta_{3}\left[x_{1} x_{2}-b x_{3}\right] .
\end{array}\right.
$$

Theorem For given constant scaling matrices $\alpha_{i}(i=1,2,3), \beta_{i}(i=1,2,3)$, modified hybrid projective synchronization between system (1) and system (3) will occur by following controller:

$$
\left\{\begin{array}{l}
u_{1}=1 / \alpha_{1}\left(\beta_{1} x_{1}-\alpha_{1} y_{1}+\beta_{1}\left(\tilde{a}\left(x_{2}-x_{1}\right)\right)-\tilde{d}\left(y_{2}-y_{1}\right)\right. \\
u_{2}=1 / \alpha_{2}\left(\beta_{2} x_{2}-\alpha_{2} y_{2}+\beta_{2}\left((\tilde{c}-\tilde{a}) x_{1}-x_{1} x_{3}+\tilde{c} x_{2}\right)+y_{1} y_{3}-\tilde{h} y_{2}\right. \\
u_{3}=1 / \alpha_{3}\left(\beta_{3} x_{3}-\alpha_{3} y_{3}+\beta_{3}\left(x_{1} x_{2}-\tilde{b} x_{3}\right)+\tilde{f} y_{3}-y_{1} y_{2},\right.
\end{array}\right.
$$

and all the parameter update rule for unknown parameters $a, b, c, d, h, f$

Combining (6) with (7), one has

$$
\left\{\begin{array}{l}
D_{*}^{q} \tilde{a}=\beta_{1}\left(x_{2}-x_{1}\right) e_{1}+\beta_{1} x_{1} e_{2} \\
D_{*}^{q} \tilde{b}=\beta_{3} x_{3} e_{3} \\
D_{*}^{q} \tilde{c}=\beta_{2}\left(x_{1}+x_{2}\right) e_{2} \\
D_{*}^{q} \tilde{d}=\alpha_{1}\left(y_{1}-y_{2}\right) e_{1} \\
D_{*}^{q} \tilde{h}=-\alpha_{2} y_{2} e_{2} \\
D_{*}^{q} \tilde{f}=\alpha_{3} y_{3} e_{3},
\end{array}\right.
$$

Proof: From Eqs.(5)-(7), we can obtain the error dynamical system as below

$$
\left\{\begin{array}{l}
D_{*}^{q} e_{1}=\alpha_{1}\left(y_{2}-y_{1}\right) e_{d}-\beta_{1}\left(x_{2}-x_{1}\right) e_{a}-e_{1} \\
D_{*}^{q} e_{2}=\alpha_{2} y_{2} e_{h}-e_{2}+\beta_{2} e_{c}\left(x_{2}+x_{1}\right)-\beta_{2} x_{1} e_{a} \\
D_{*}^{q} e_{3}=\alpha_{3} y_{3} e_{f}-\beta_{3} x_{3} e_{b}-e_{3}
\end{array}\right.
$$

$$
\begin{aligned}
J= & e_{1} D_{*}^{q} e_{1}+e_{2} D_{*}^{q} e_{2}+e_{3} D_{*}^{q} e_{3}+e_{a} D_{*}^{q} e_{a}+e_{b} D_{*}^{q} e_{b} \\
& +e_{c} D_{*}^{q} e_{c}+e_{d} D_{*}^{q} e_{d}+e_{h} D_{*}^{q} e_{h}+e_{f} D_{*}^{q} e_{f} .
\end{aligned}
$$


From Eq.(5-8), we can get that

$$
\begin{aligned}
& e_{1} D_{*}^{q} e_{1}+e_{2} D_{*}^{q} e_{2}+e_{3} D_{*}^{q} e_{3}+e_{a} D_{*}^{q} e_{a}+e_{b} D_{*}^{q} e_{b}+e_{c} D_{*}^{q} e_{c}+e_{d} D_{*}^{q} e_{d}+e_{h} D_{*}^{q} e_{h}+e_{f} D_{*}^{q} e_{f} \\
& =e_{1}\left[\alpha_{1}\left(y_{2}-y_{1}\right) e_{d}-\beta_{1}\left(x_{2}-x_{1}\right) e_{a}-e_{1}\right]+e_{2}\left[\alpha_{2} y_{2} e_{h}-e_{2}+\beta_{2} e_{c}\left(x_{2}+x_{1}\right)-\beta_{2} x_{1} e_{a}\right]+e_{3}\left[\alpha_{3} y_{3} e_{f}-\beta_{3} x_{3} e_{b}-e_{3}\right] \\
& +e_{a}\left[\beta_{1}\left(x_{2}-x_{1}\right) e_{1}+\beta_{1} x_{1} e_{2}\right]+e_{b}\left[\beta_{3} x_{3} e_{3}\right]+e_{c}\left[\beta_{2}\left(x_{1}+x_{2}\right) e_{2}\right]+e_{d}\left[\alpha_{1}\left(y_{1}-y_{2}\right) e_{1}\right]+e_{h}\left[-\alpha_{2} y_{2} e_{2}\right]+e_{f}\left[\alpha_{3} y_{3} e_{3}\right] \\
& =-e_{1}^{2}-e_{2}^{2}-e_{3}^{2} \leq 0 .
\end{aligned}
$$

Then the response system (3) can synchronize the drive system (1) globally and asymptotically.

\section{Numerical simulations}

We choose the scaling matrices $\alpha=\operatorname{diag}(1,1,1), \beta=\operatorname{diag}(-2,-2,-2)$. The values of unknown parameters converge to $\tilde{a}=35, \tilde{b}=3, \tilde{c}=28, \tilde{d}=36, \tilde{h}=20, \tilde{f}=3$ is shown in Fig. 4.
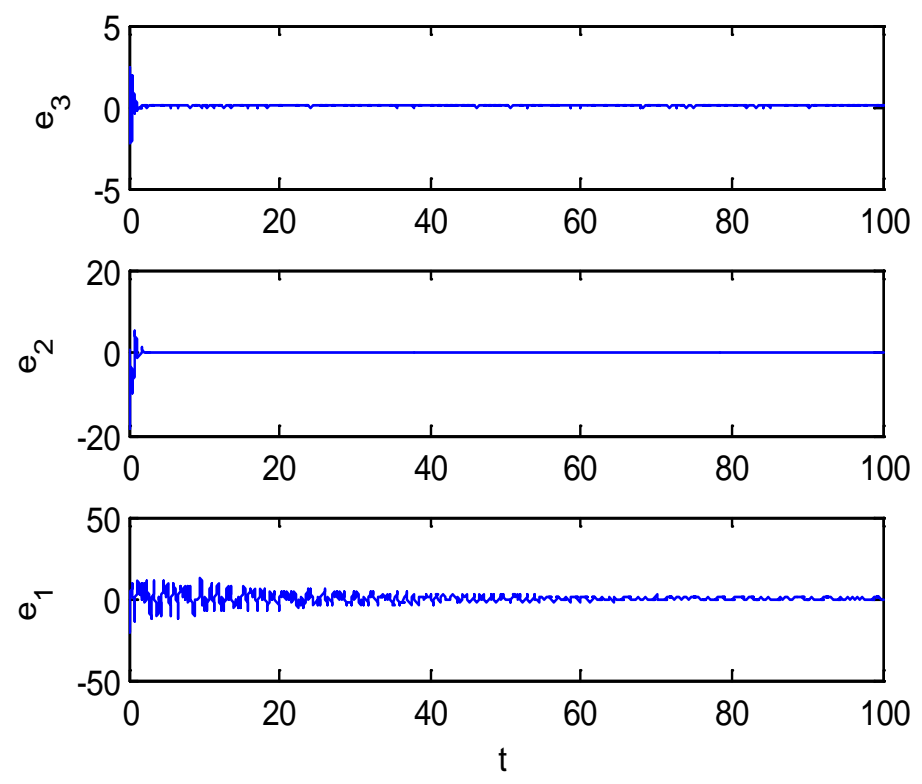

Fig.2. Errors of drive and response system with $\alpha=\operatorname{diag}(1,1,1), \beta=\operatorname{diag}(-2,-2,-2)$
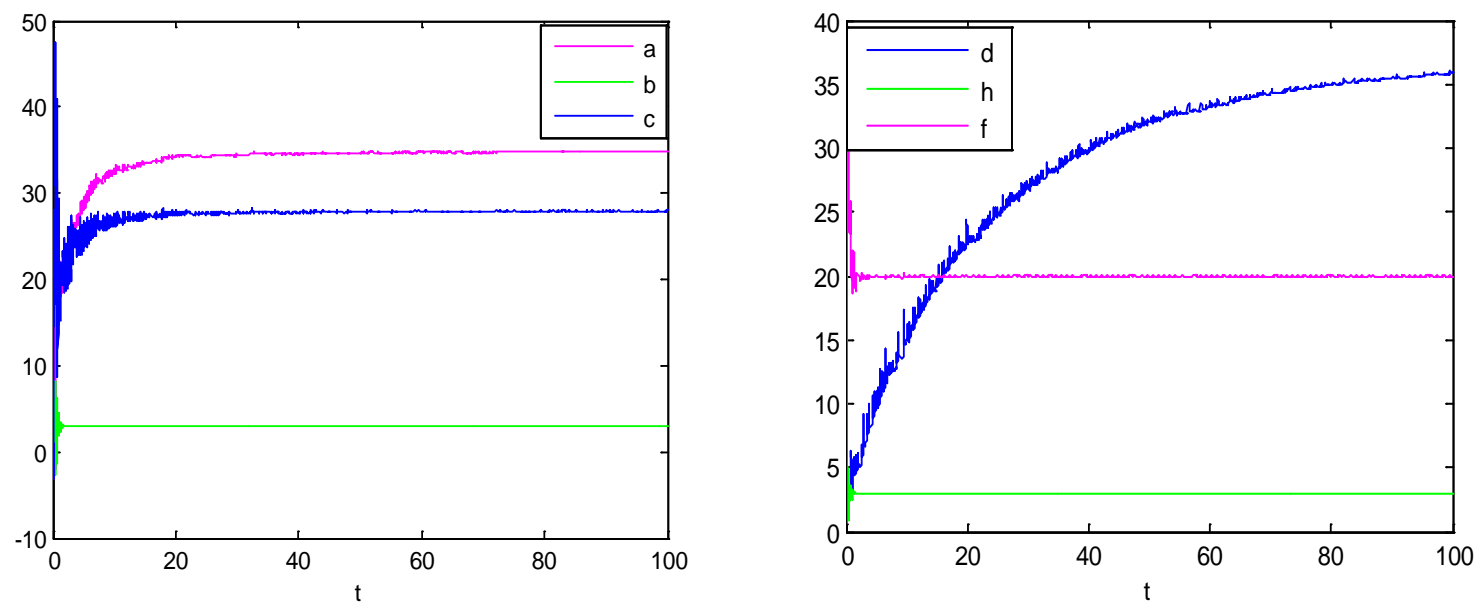

Fig.3. Identification curves of the unknown parameters

\section{Conclusions}

Based on the stability theory of fractional-order system, adaptive controller and parameter update law are designed to ensure Chen system synchronize with Lü system up to double scaling matrices. Numeric results show that the proposed scheme is analytically rigorous and practically feasible. 


\section{References}

[1] I.Podlubny, Fractional Differential Equations, Academic Press, New York,1999.

[2] L.M. Pecora, T.L. Carrol: Synchronization in chaotic systems. Phys. Rev. Lett. 64(1990) 821-826.

[3] W.H. Deng, C.P. Li: Chaos synchronization of the fractional Lü system. Physica A. 353 (2005) 61-69.

[4] C.P. Li, W.H Deng, and D. Xu: Chaos synchronization of the Chua system with a fractional order. Physica A, 360(2006): 171-185.

[5] J.H. Lu: Synchronization of a class of fractional-order chaotic systems via a scalar transmitted signal. Chaos Solitons \& Fractals. 27 (2006) 519-526.

[6] C.M. Chang, H.K. Chen: Chaos and hybrid projective synchronization of commensurate and incommensurate fractional-order Chen-Lee systems. Nonlinear Dyn.62 (2010)851-863. 